\title{
Propuesta de formación guiada con dinámica de sistemas para promover el desarrollo de software orientado por modelos en sistemas productivos rurales que requieren asociatividad
}

\author{
Urbano E. Gómez-Prada \\ Facultad de Ingeniería de Sistemas e Informática, Universidad Pontificia Bolivariana, Bucaramanga, Colombia. \\ (Correo-e: urbano.gomez@upb.edu.co)
}

Recibido Jul. 7, 2021; Aceptado Sep. 21, 2021; Versión final Oct. 25, 2021, Publicado Feb. 2022

\begin{abstract}
Resumen
Este estudio tiene como objetivo desarrollar una propuesta de formación que ayuda a comprender la complejidad de los sistemas productivos rurales e inferir la importancia de la asociatividad. La propuesta adopta la dinámica de sistemas para el modelamiento semántico en el desarrollo de software integrando temáticas de las asignaturas de pensamiento sistémico y de diseño. La propuesta es aplicada siguiendo un diseño cuasi-experimental con dos grupos (control y experimental) en el programa de ingeniería de sistemas e informática de la Universidad Pontificia Bolivariana (UPB) de Colombia. Los resultados muestran que los estudiantes mejoraron la comprensión en un $38 \%$ en el modelo del fenómeno físico, un $29 \%$ en el modelo de la oferta y la demanda y un $23 \%$ en el modelo de producción bovina. Se concluye que la propuesta de formación ayuda a diseñar software para sistemas productivos rurales, así como a comprender su complejidad y la importancia de la asociatividad en ellos.
\end{abstract}

Palabras clave: formación guiada; software orientado; modelos; producción rural; dinámica de sistemas

\section{System dynamics training proposal to promote model-oriented software development in rural productive systems that require associativity}

\begin{abstract}
The current study develops a training proposal that aims to facilitate the understanding of rural production systems while assessing the role and importance of associativity. The proposal adopts the System Dynamics for semantic modeling in the software development processes integrating content from systemic thinking and design courses. The proposal is applied by following a quasi-experimental design with two groups (control and experimental) of students enrolled in the systems engineering and informatics program at the Universidad Pontificia Bolivariana (UPB) in Colombia. The results show that students' performance improved their comprehension by $38 \%$ in the physics phenomenon model, by $29 \%$ in the demand and supply model, and $23 \%$ in the bovine production model. It is concluded that the proposal developed helps to design software for rural production systems as well as to understand its complexity and the importance of associativity in them.
\end{abstract}

Keywords: guided training; model-driven software; rural production; system dynamics 


\section{INTRODUCCIÓN}

Un sistema según Bunge (2018) es un conjunto de componentes con propiedades específicas y analizables interrelacionados entre sí y con el ambiente en el que se encuentra se definen límites, condiciones, recursos e información que inciden en él y que generan su estructura y sus funciones. Los sistemas se deben conocer para generar resultados positivos sobre las decisiones a tomar sobre problemas que se pueden presentar, y su conocimiento requiere un aprendizaje que lleve al reconocimiento de modelos mentales sobre ellos, pero, éste aprendizaje exige que sea significativo ya que es necesario permitir que la realimentación influya en los modelos y los transforme en una mejor aproximación de la realidad (O'Connor y McDermott, 1998).

De un sistema cada persona tiene una comprensión conceptual, es decir, una representación del modelo mental, en donde, según el conocimiento que se tenga del sistema, servirá o no, como aproximación de la realidad. Este modelo se puede formalizar en algún lenguaje y existen algunos que permiten simular el comportamiento de variables del sistema y con ello brindan la posibilidad de que quien los construye y quien los interpreta, generen experiencias que mejoran la comprensión del sistema y se usen para el análisis y la toma de decisiones (Markauskaite et al., 2020). Al respecto, Senge et al. (2001) manifiestan que los modelos son laboratorios de aprendizaje asistidos por un simulador informático para construir lo comprendido de una situación y experimentar estrategias de los aspectos planteados. En un sistema se deben tomar decisiones periódicas por parte de quien lo administra y requiere, además del modelo, de un software que las apoye. Un software es un conjunto de componentes orientados a la gestión de los datos generados, estos componentes están organizados para cumplir el objetivo de presentar información y adquiere complejidad dependiendo de su tamaño y las interacciones del sistema para el que fue construido y cuando su desarrollo es guiado por modelos, se mejora la productividad y la calidad del producto, y se reduce el tiempo que toma la fase de programación (Brambilla et al., 2017).

La propuesta de formación está dirigida a futuros desarrolladores de software que cursan Pensamiento Sistémico y Diseño de Software y, tiene las intenciones de dar a conocer: i) la complejidad que se da en los sistemas de producción; complejidad que se da por las interacciones entre los elementos del sistema (De Buck et al., 2001) y que debe ser comprendida para que, los estudiantes en sus desarrollos, le faciliten al usuario la toma de decisiones a partir del control en la gestión de los procesos, ii) la Dinámica de Sistemas como elemento para comprender el diseño de software, iii) la importancia el desarrollo de software orientado por modelos y iv) los beneficios productivos que trae la asociatividad como el de mitigar brechas en los precios y optimizar el uso de recursos, una necesidad descrita por autores citados por Gómez et al. (2019).

La Dinámica de Sistemas es una metodología para representar sistemas mediante modelos de simulación que en esta propuesta son elaborados en el entorno llamado Evolución (Lince et al., 2011) y puede aumentar la eficacia de la ingeniería al mejorar la comprensión de sistemas por usar modelos de simulación como artefactos de utilidad (Senge, 2001) que en este caso apoyan el desarrollo de software para el modelamiento semántico, una posibilidad que otros autores ya han considerado. Algunos de estos son: i) Tulinayo et al. (2011) se apoyaron en el modelado para desarrollar un software que apoya el proceso de gestión hospitalaria para mujeres embarazadas desde el ingreso hasta su dada de alta. ii) Jaime (2012) simuló un sistema productivo de conejos y sus requisiciones de alimento y mostró que la Dinámica de sistemas facilita la representación del conocimiento en el dominio del problema, la comunicación entre clientes y desarrolladores en la construcción y la validación del software. iii) Gómez \& Gómez (2013) desarrollaron un software para la administración de empresas de producción textil en donde su fase de análisis y diseño fue soportada en un modelo en Dinámica de Sistemas con elementos como clientes, inventarios, productos, insumos, pedidos de clientes y de proveedores, órdenes de compra, mano de obra, maquinaria, ventas, devoluciones, entre otros. iv) Liebet et al. (2014) encontraron, tras analizar 112 áreas en donde trabajan desarrolladores de Medicina basada en la evidencia, que se requiere un gran esfuerzo de formación para que se comprendan los procesos y desarrollen buenas interfaces que ayuden a que se use el software.

El modelamiento semántico permite representar el software y en esta propuesta su representación se apoya en lenguajes de la Dinámica de Sistemas con los que se generan modelos en un proceso de cobertura creciente para facilitar la comprensión. Los lenguajes según Andrade et al. (2001) son: i) El lenguaje de la Prosa el cual es para hacer la descripción del sistema en lenguaje natural y con ello expresar la comprensión que se tiene e identificar las variables y las relaciones que lo componen. ii) El lenguaje de las influencias es un esquema donde se relacionan con flechas, las variables identificadas, mostrando la influencia de esa variable con la que está en el destino, la combinación de varias relaciones de influencia puede generar ciclos de realimentación que indican lo dinámico del sistema. iii) El lenguaje de Flujo-Nivel es una representación basada en elementos propios de la metodología que aluden a la variación en función del tiempo de algunas variables y representan la lógica matemática. Otros elementos en este leguaje son las auxiliares, retardos, multiplicador, entre otros. iv) El lenguaje de las ecuaciones en notación Euler es una representación que requiere de un software específico para su evaluación en cada paso de simulación. v) El lenguaje de los comportamientos simulados presenta los valores obtenidos por cada variable en función del tiempo tras la evaluación de las ecuaciones. 
La Dinámica de Sistemas además de permitir la construcción de modelos de simulación es usada para soportar la construcción de diagramas UML por su facilidad para apoyar la comprensión de sistemas. Las reglas para usarla en el modelamiento semántico, son: i) El nivel representa una clase o un atributo de una clase, ii) el flujo es un método iii) la relación define una relación entre clases. iv) si las clases comparten métodos se pueden unir o denotar herencia y conviene marcarlas con un sector para dar claridad, 5) Los retardos y demás elementos denotan la definición de métodos y apoyan el diseño del diagrama de actividades.

En la Figura 1 es presentado como ejemplo, una parte del modelo de una finca ganadera que se aplica para realizar el modelado semántico. En la parte a se aprecia el diagrama de influencias, en la parte b, el lenguaje de Flujo-Nivel correspondiente, y en c, el diagrama de clases generado a partir de la semántica propuesta. Observe que los niveles se agruparon en la clase Animal que toma como métodos los flujos y la clase Producto que representa la leche que se da en el sistema a una tasa de producción indicada por el parámetro; en el diagrama de Flujo-Nivel hay elementos propios de este lenguaje que no están en la parte a y la parte c, pero se colocaron en la figura asignándoles el mismo nombre del elemento que representan, para dar a conocer otros elementos de la Dinámica de Sistemas). Además, en a hay relaciones de color negro que, al pasarlas a b, son flujos y su símbolo allí es una flecha negra y una válvula. La variable VDD corresponde a las vacas en descanso que aumentan cuando hay partos, y disminuyen al finalizar el periodo de lactancia. VP son las vacas preñadas que aumentan cuando se insemina o se desteta una vaca, y disminuye cuando dan a luz. El parámetro es el promedio de producción de leche por animal, es decir, "ppla" en el la parte (c).

(a)

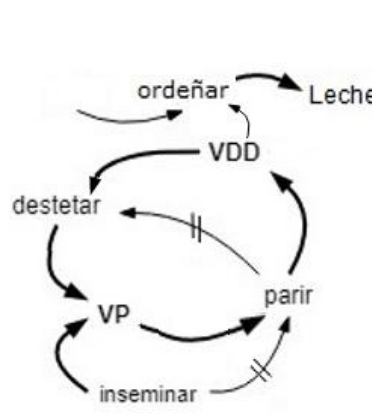

(b)

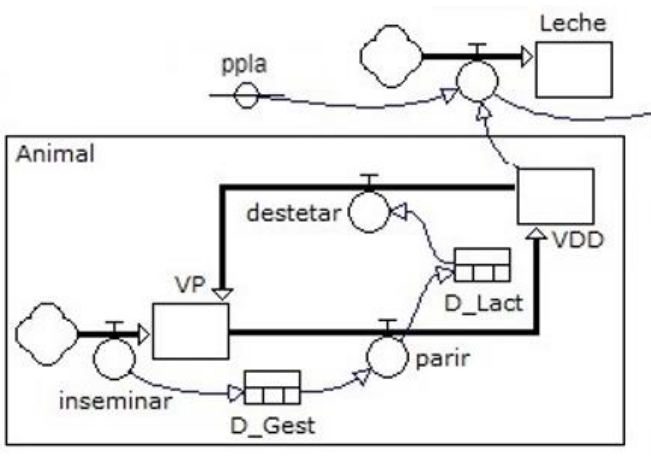

(c)

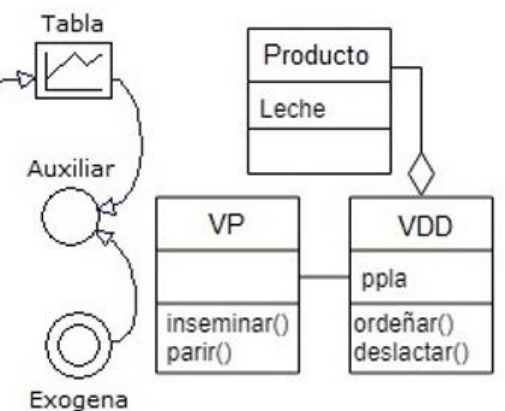

Fig. 1. Estructura Básica del modelo en el lenguaje de Flujo-Nivel

En este lenguaje, cada flujo conectado a un nivel representa una ecuación diferencial que corresponde a la ecuación 1 (en la figura hay tres ecuaciones diferenciales, pero solo se presenta una en este documento). La ecuación es transformada a modo Euler en la ecuación 2 y a notación infinitesimal en la ecuación 3. La ecuación 2 indica que el nivel en un nuevo paso de tiempo $\Delta t$, se obtiene sumando el valor del nivel y flujo que existían en el tiempo anterior; el paso es asumido constante durante el período de tiempo, es decir, indica cómo varia el nivel en función del flujo en los pasos o intervalos de tiempo. Los demás elementos también simbolizan ecuaciones evaluadas en cada paso de tiempo para generar el comportamiento de cada variable, pero no corresponde a ecuaciones diferenciales como la expresada en 1, 2 y 3 . Además de flujos y niveles, hay otros elementos descritos en términos de Parámetros, Retardos o Demoras, Multiplicadores, Auxiliares y Exógenas (también hay Sectores, Subsistemas y Elementos de lógica difusa).

$$
\begin{aligned}
& \frac{d(V D D)}{d t}=\operatorname{parir}(t)-\operatorname{destetar}(t) \\
& V D D(t+\Delta t)=(V D D(t)+\operatorname{parir}(t)-\operatorname{destetar}(t)) * \Delta t \\
& V D D_{t}=V D D_{t-1}+\operatorname{parir}_{t-1}-\text { destetar }_{t-1}
\end{aligned}
$$

En la propuesta se explican cuatro situaciones con modelos de simulación para buscar un aprendizaje significativo, el cual requiere estrategias donde los estudiantes tengan la posibilidad de representar el sistema y simular varios escenarios tal y como lo propone Sánchez (2012). En resumen, las cuatro situaciones son: i) Movimiento rectilíneo uniformemente acelerado, el cual es aquel en el que un auto se desplaza sobre una trayectoria recta estando sometido a una aceleración que puede ser constante o variable y que fue descrito por Gómez et al. (2017) al presentar un simulador de robots seguidores de línea en el que estudiantes de ingeniería desarrollan los algoritmos que controlan el robot y los prueban simulando una competencia. ii) Oferta y Demanda: Representa la variación que se da por la relación entre la demanda de un producto y la cantidad ofrecida a partir del precio al que se vende, por ello, el precio en el mercado del producto influencia la cantidad de productos a fabricar y la cantidad que los compradores están dispuestos a comprar (Vera et al., 2019). El modelo orienta sobre como buscar este equilibrio al orientar sobre las razones de cambio de los 
precios de los productos que se dan por las fluctuaciones en la producción. El modelo simula escenarios para el agro, sector que tiene la necesidad de mitigar brechas de precios y que ya se ha buscado en herramientas como el software Agrocraft, el cual anuncia la demanda futura y provee a productores rurales asociados, la oferta a generar (Gómez et al., 2016). iii) Producción bovina: Representa un sistema llamado finca, una organización con procesos de producción y comercialización de carne y leche que cuenta con recursos demográficos, biofísicos, productivos y financieros que se deben controlar para generar utilidades (Gómez et al., 2015). iv) Integración de la Oferta y la Demanda con la producción bovina: Representa la integración del segundo y el tercer modelo realizado al que le fueron agregados los subsistemas de agua y suplemento.

\section{METODOLOGÍA}

La propuesta para promover el desarrollo de software orientado por modelos en sistemas productivos rurales se soporta en cuatro modelos de simulación elaborados con Dinámica de Sistemas, los cuales deben ser comprendidos por los estudiantes de Pensamiento Sistémico y Diseño de Software. Fue ejecutada siguiendo un diseño cuasi-experimental con dos grupos (uno experimental y uno de control, conformados por estudiantes de Ingeniería de Sistemas de primer semestre), con quienes se siguieron las siguientes fases: i) Medir conocimientos o pre-saberes sobre cada sistema. Para ello se aplicó un instrumento con preguntas sobre los sistemas de Movimiento Rectilíneo, Oferta y Demanda y Producción bovina. ii) Explicar los sistemas de manera teórica. iii) Presentar, al grupo experimental, los modelos soportados en Dinámica de Sistemas permitiendo su revisión y simulación para varios escenarios (al grupo de control no se le aplica esta fase). iv) Evaluar el aprendizaje sobre los sistemas en cada grupo mediante un instrumento con preguntas similares a las de la primera fase. v) Diseñar un diagrama del software en UML que sirva como ambiente virtual de aprendizaje sobre el sistema productivo que integra varias fincas e incluya, la oferta y la demanda, actividad que se apoya en la presentación como antecedente de un videojuego serio de ganadería bovina desarrollado en una estrategia de apropiación desarrollado por Gómez et al., (2020), el cual es un ejemplo con el que los estudiantes visualizan la idea de la tarea asignada. vi) Homogenizar el conocimiento del sistema presentando los modelos al grupo de control para también tomar conciencia sobre la complejidad en algunos procesos.

\section{RESULTADOS Y DISCUSIÓN}

En la propuesta se miden los conocimientos sobre los sistemas de interés, se presentan la explicación de los sistemas y los modelos desarrollados, se mide el aprendizaje y, utilizando el último modelo, se generan los diagramas UML para que dirijan la construcción de un prototipo software propuesto por cada estudiante.

\section{Medición de saberes previos y posteriores a la explicación}

Según la metodología propuesta, en la primera y cuarta fase se miden saberes sobre los sistemas y tras aplicar la Dinámica de Sistemas se comparó el aprendizaje con instrumentos que tenían preguntas similares. En la Figura 2 son presentados los resultados. En la parte a, se aprecian los valores obtenidos para los tres sistemas de la propuesta con el porcentaje de aciertos obtenido antes y después del experimento para el grupo experimental y del control. En la parte b se presenta el contraste de resultados para cada sistema entre los dos grupos, en donde se aprecia que en el modelo del fenómeno físico mejoraron en un $38 \%$, en la comprensión del modelo de la oferta y la demanda un $29 \%$ y en el modelo de producción bovina un $23 \%$.

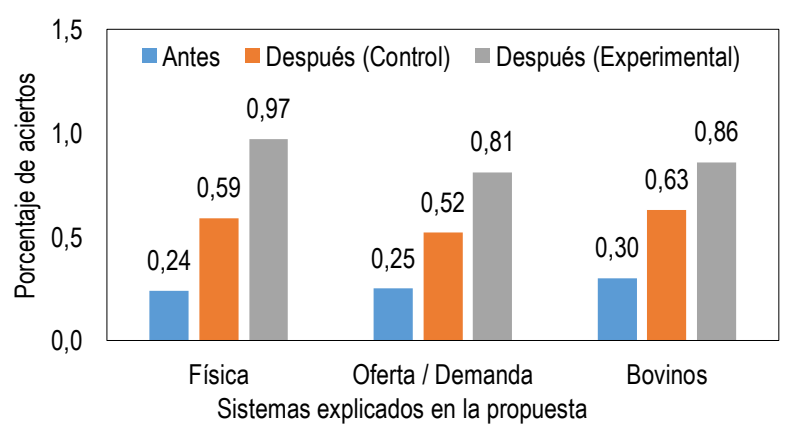

(a)

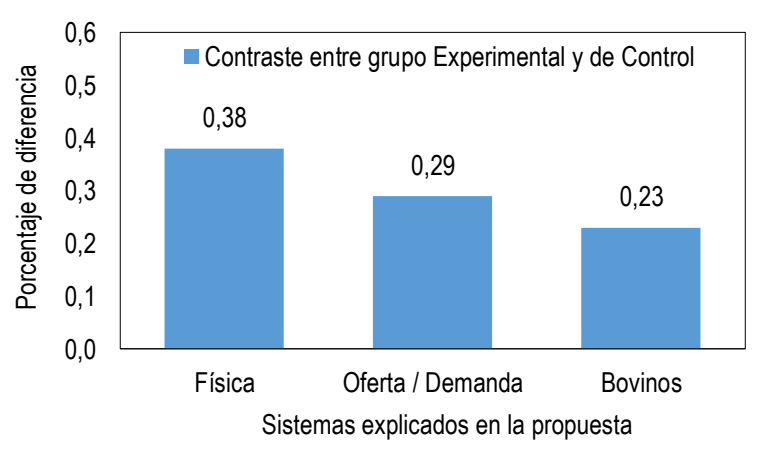

(b)

Fig. 2. Resultados de la comparación de aprendizaje en los sistemas de estudio

\section{Presentación de los modelos (fases ii y iii)}

La propuesta continua con la presentación de los modelos permitiendo a los estudiantes reflexionar sobre la estructura y simular escenarios. El primer modelo debe ser sencillo ya que se utilizó para reforzar el tema de Dinámica de Sistemas. En este caso se modeló el movimiento rectilíneo con y sin aceleración. La explicación del sistema inicia con la conceptualización, continuando con la construcción del diagrama de influencias. 
Luego se hace el de flujo de nivel y se presentan las ecuaciones con su evaluación con lo cual se van generando los comportamientos y después, se aumenta la complejidad hasta alcanzar el modelo presentado en la figura 3, el cuál es el tercer prototipo y tiene el diagrama de influencias, debajo está el diagrama de FlujoNivel y a la izquierda se presentan los comportamientos simulados. Durante la actividad se les pide a los estudiantes responder preguntas sobre los valores que se deben ir dando, en cada unidad de tiempo, la posición, la velocidad o la aceleración, a partir de diferentes valores entre estos.

El modelo propuesto para comprender la variación de la Oferta y la Demanda en un sistema productivo es presentado en la figura 4 en el lenguaje de Flujo-Nivel y en uno de los comportamientos. En el modelo hay dos niveles que representan el inventario de un producto y se aprecian las siguientes influencias: (1) El inventario varía según la producción y los despachos. (2) El precio varía en función de la relación entre el inventario y la Demanda. (3) El precio tiene un efecto en la producción y en la demanda que es generada por los despachos. La relación entre el inventario y la demanda genera variaciones de inestabilidad en el precio generando oscilaciones en la producción y los despachos, el del precio y la producción se aprecia en la figura. El modelo del sistema de producción bovina involucra recursos demográficos, biofísicos, productivos y financieros y se tomó como base el presentado por Gómez et al. (2015) y hace parte de la integración realizada con el modelo de la oferta y la demanda por tanto su explicación se dará en conjunto a continuación.
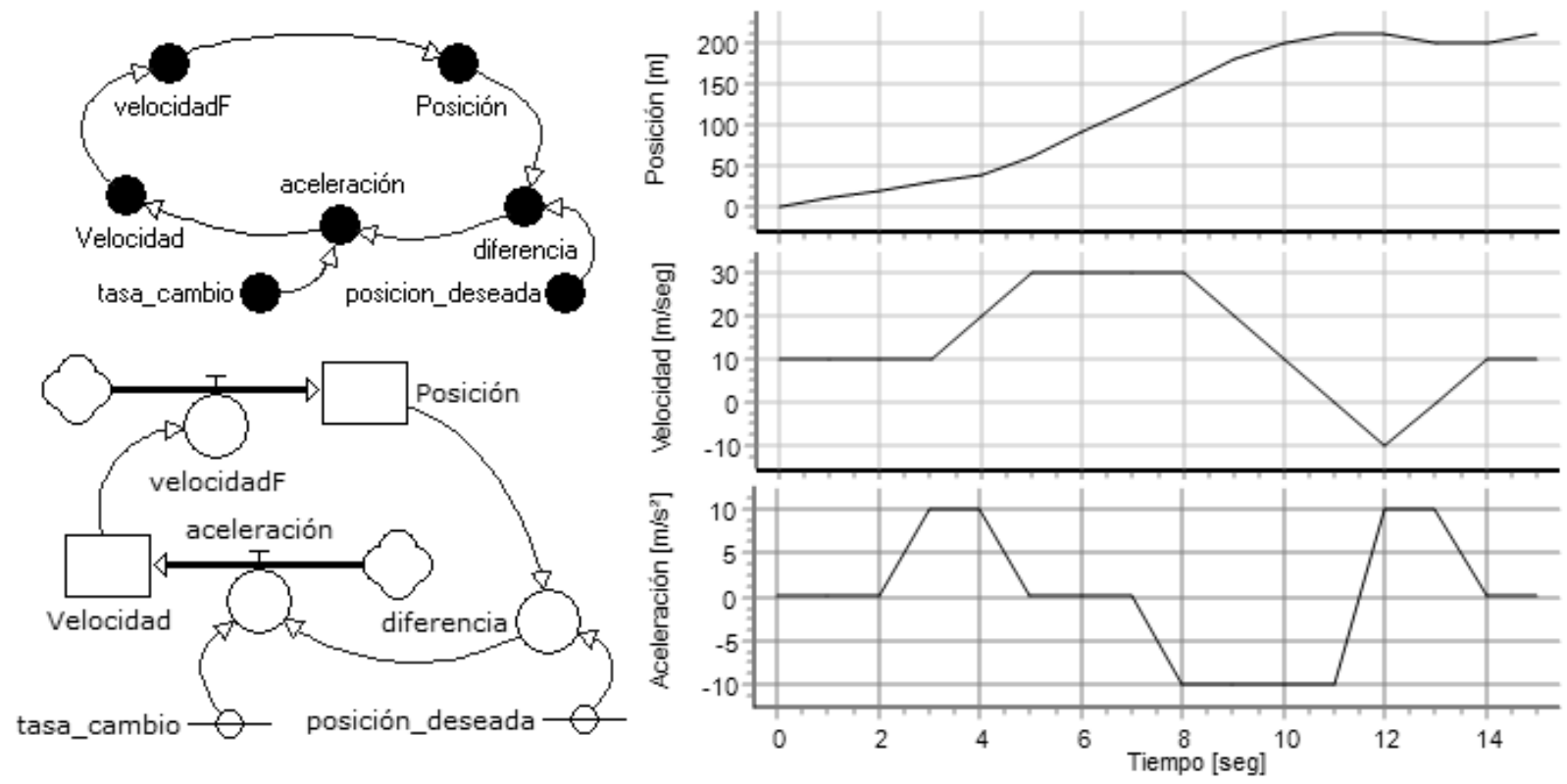

Fig. 3. Ejemplo del modelo de movimiento rectilíneo
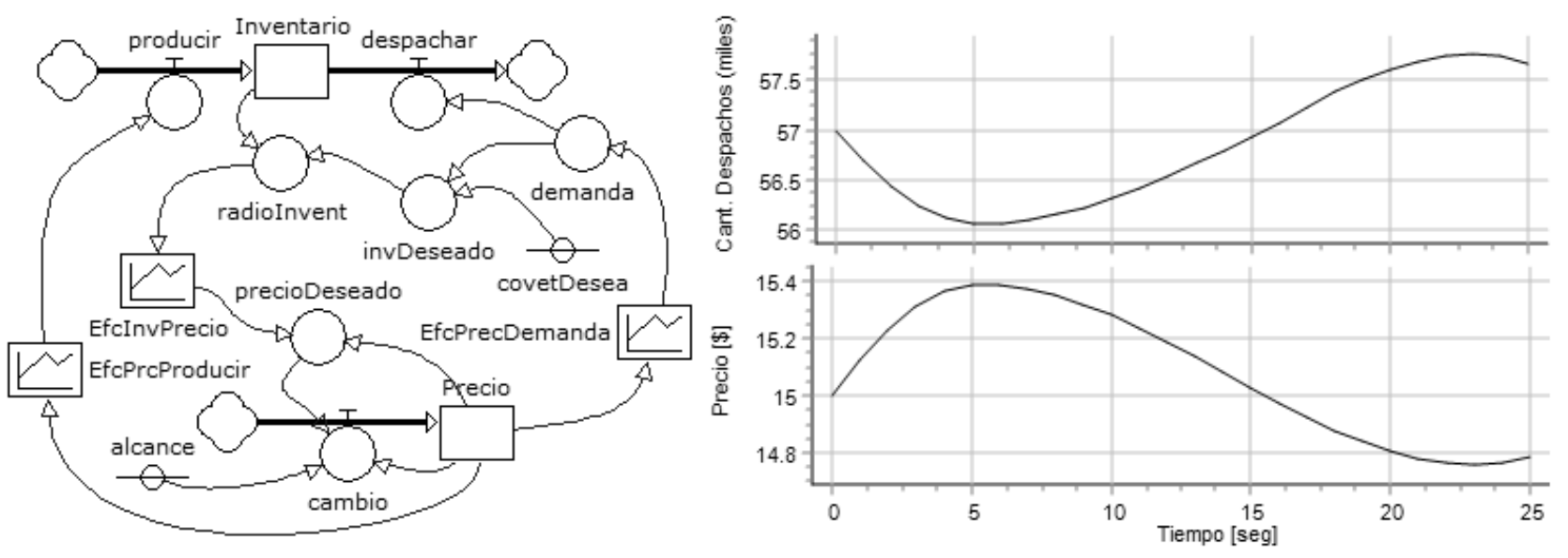

Fig. 4. Ejemplo del Modelo de Oferta y Demanda

Tras comprender la complejidad de una finca se procede a mostrar la importancia de la asociatividad ya que se debe entender que cuando varias fincas generan productos o compran suplemento o utilizan recursos, el sistema adquiere aún más complejidad y, si se produce en exceso y se desconoce lo que los demás producen, se puede llevar al exceso de producción y, por tanto, a una disminución de precios. Además, se puede agotar el suplemento o el agua por no conocer los requerimientos de los demás; frente a esta necesidad, podría implementarse también el modelo de Oferta/Demanda para la fabricación de suplemento). La figura 5 presenta 
la estructura cuando hay interacción de varias fincas; en el esquema las fincas comercializan sus productos y compran el suplemento en un mismo lugar y que todas adquieren el agua de la misma fuente.

La integración del modelo de la Oferta y Demanda con el modelo de producción bovina surge el deseo de querer demostrar que la asociatividad entre los productores mejora para ellos las condiciones de mercado y el uso de los recursos ambientales comunes. El modelo presenta cuatro sectores integrados que son pertinentes al administrar la finca: la finca, la Oferta y Demanda, la fabricación de suplemento y el agua. Las interacciones entre los cuatro sistemas muestran que: (1) es preciso un uso racional del agua y del suplemento ya que es usual que no se considere su costo en los sistemas reales, por ello se incluyeron recursos comunes, son recursos expuestos a la explotación excesiva causada por el interés individual que lleva a su destrucción (Hurnyak y Kordonska, 2018) y (2) si se produce en exceso, hay disminución del precio de venta por desconocer la demanda. Por ello se involucra la asociatividad, asumida como la acción de generar grupos de personas que sumen esfuerzos para compartir ideales y den respuestas colectivas de colaboración donde surjan acciones para afrontar necesidades de mercados globales (Fonseca et al., 2020).

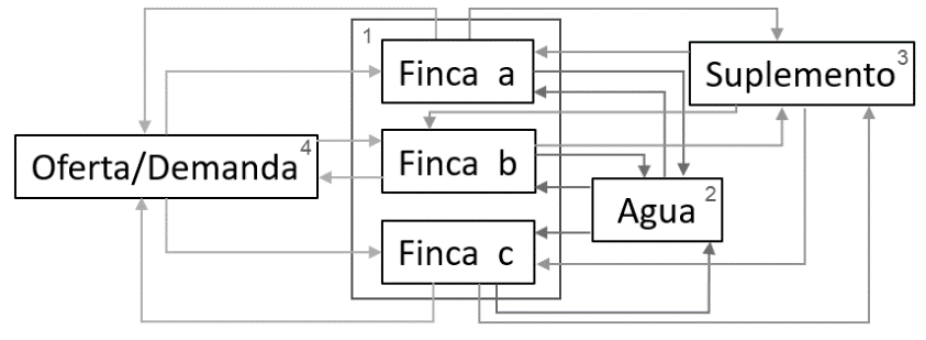

Fig. 5. Estructura del sistema con la interacción de varias fincas centralizadas

En la figura 6 se aprecia entre rectángulos llamados sectores, elementos que componen un nuevo modelo que representa una finca, la oferta y demanda, el agua y la fábrica de suplemento. La interacción de los elementos genera varios ciclos de realimentación de los cuales se van expresando uno a uno en las sesiones hasta que se comprende el sistema completo. En la figura se marcaron seis ciclos representativos pues no se pueden marcar todos por la complejidad del sistema. Aplicando la semántica propuesta, la negrilla en las palabras evidencia variables de tipo nivel que corresponde a entidades o atributos, y las fechas más gruesas denotan flujos es decir métodos de la clase que relacionan. Observe que dentro de la finca hay un rectángulo que contiene los animales y representa cómo, en función a estos, se calcula el consumo de agua y de suplemento. Para facilitar la comprensión se quitaron elementos como los animales machos, las estructuras que representan el crecimiento y la producción de leche y no están los elementos y los retaros que denotan los tiempos necesarios para la transformación de vacas preñadas (VP) a vacas en descanso (VDD) o para que el suplemento pase de estar en producción (InvPrd) a estar disponible para su adquisición (InvAlm).

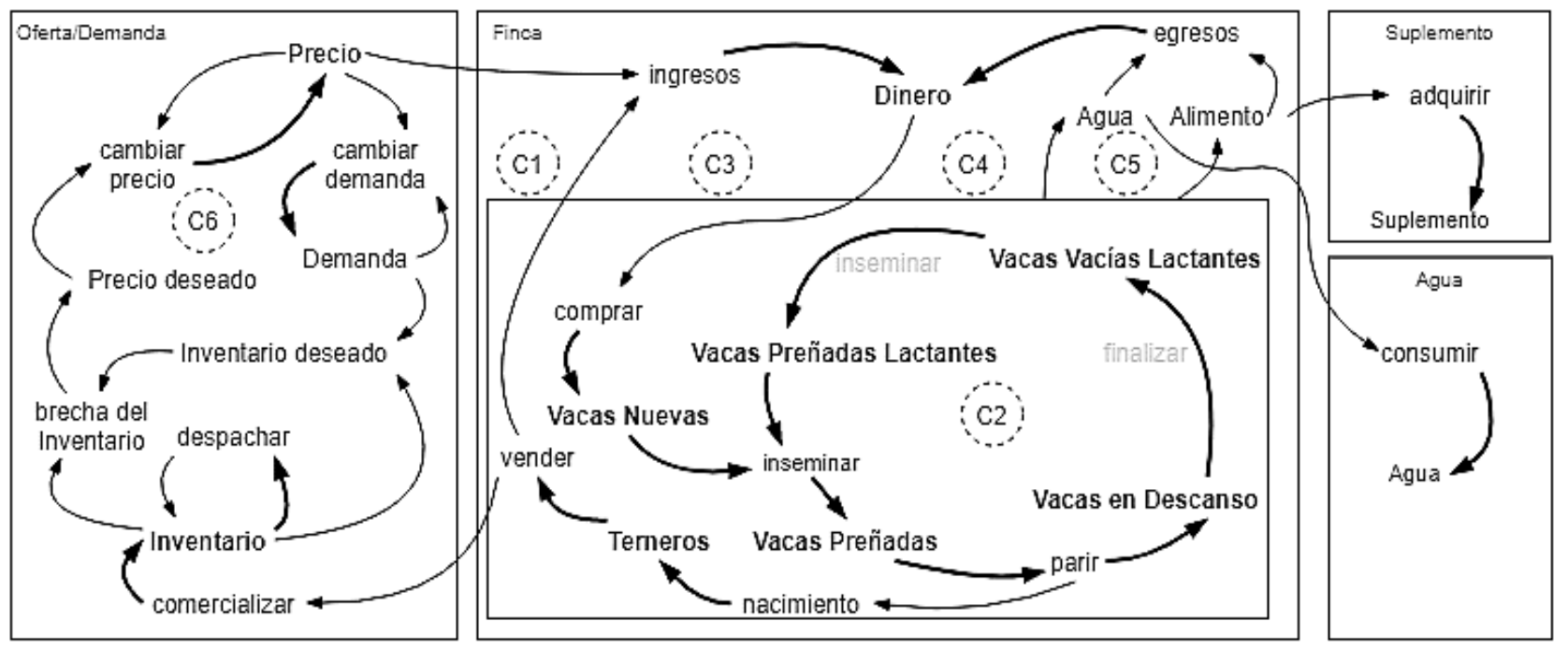

Fig. 6. Lenguaje de influencias resumido del modelo integrado

El ciclo 1 se genera al vender productos y estas ventas generan ingresos de capital (dinero) con el cual se puede comprar animales e insumos para generar productos como leche y carne que se vende. El ciclo 2 se da en el componente animal de la finca, el cual inicia al comprar vacas nuevo vientre, las cuales se inseminan y se convierte en vacas preñadas, las vacas preñadas, nueve meses después dan a luz a una ternera y pasa al grupo de vacas en días de descanso, cuando las vacas terminan su descanso se convierten en vacías 
lactantes, se inseminan y se convierten en preñadas lactantes. Tres meses antes del parto, las vacas se dejan de ordeñar y pasan nuevamente a ser preñadas. El ciclo 3 expresa que el Dinero depende de los ingresos y los egresos. Los ingresos se dan por la venta de terneras y de leche y los egresos por la compra de animales, el pago de la mano de obra y del alimento calculado por una proporción del peso promedio. El ciclo 4 expresa que se requiere gua que se genera al llover y su consumo se calcula por una proporción del peso total de los animales e involucra un costo que ayuda a tomar conciencia de ahorro. El ciclo 5 presenta el sector de Oferta/Demanda, es presentado el precio de venta, la Demanda, el inventario. Los últimos generan un efecto en el precio, el cual tiene un efecto en la demanda y la producción. Algunos elementos fueron reubicados por facilidad del modelo final. El ciclo 6 presenta el sector de suplemento, en este se presenta su inventario, que depende de la producción, y esta a su vez de la fabricación que se calcula en función del alimento a comprar. El modelo en lenguaje de influencias es convertido a Flujo-Nivel y una parte de este se presenta en la figura 7. Observe que el modelo presenta los mismos sectores de la figura 6 pero tiene más elementos por las necesidades propias del lenguaje, además, en algunos se redujo el nombre.

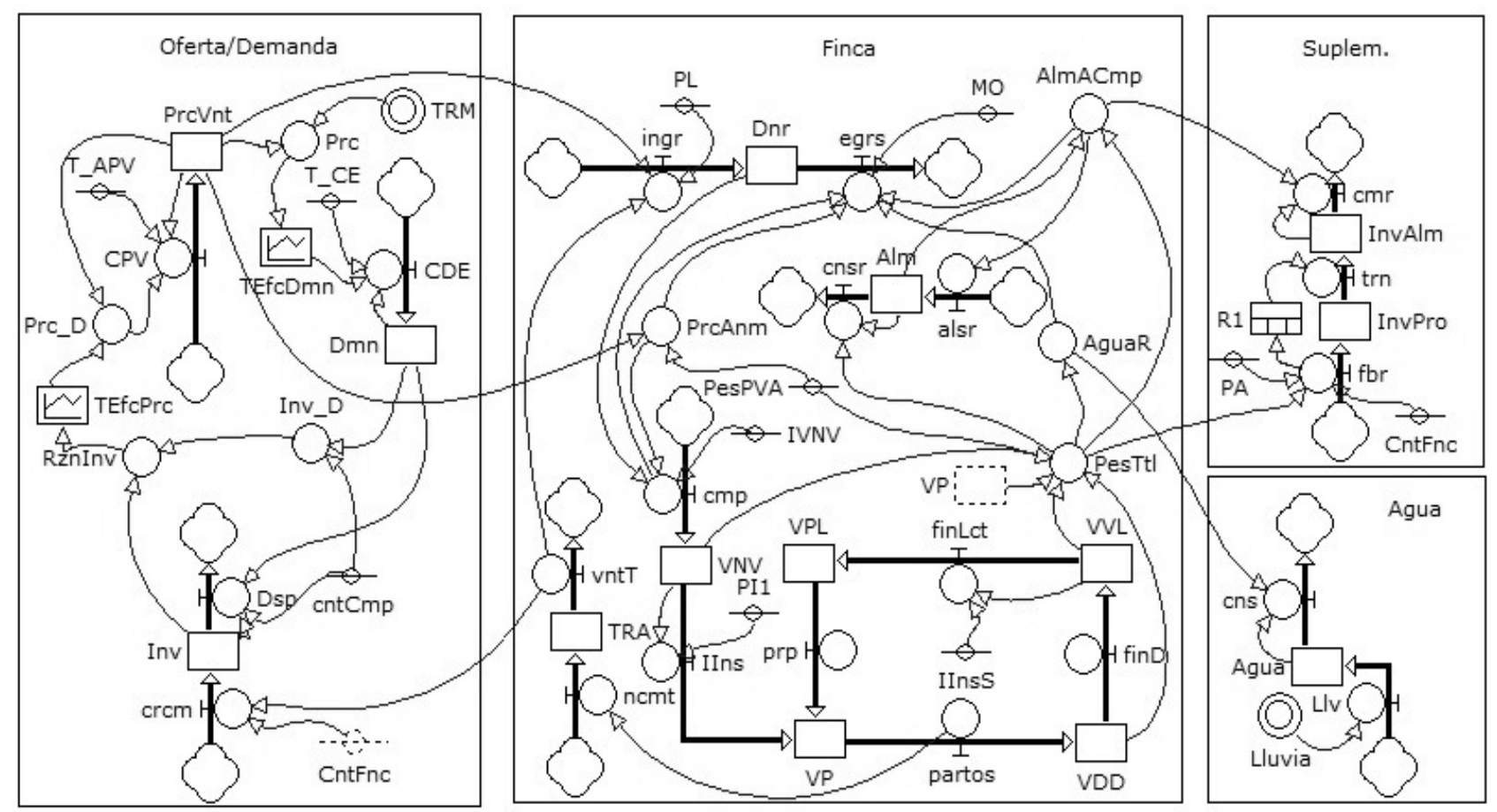

Fig. 7. Lenguaje de Flujo-Nivel integrado de Oferta y Demanda, Producción bovina, Agua y Suplemento

Al simular el modelo, el usuario debe (1) Conocer los parámetros del escenario asignado como precios por kilogramo del animal, del litro de leche, del kilogramo de suplemento, del litro de agua, de la demanda esperada, del inventario deseado, entre otro. (2) Tomar decisiones en cuanto a cuando adquirir suplemento o comprar animales para que crezcan de la mejor manera y producir carne y leche, cuándo vender algunos de sus productos o consumir agua o suplemento. (3) Ser consciente de que las decisiones generan ingresos y afectan el inventario de leche, carne, agua y suplemento, el cual, según la demanda puede generar que suba o baje el precio. (4) Conocer los valores que se generan en cada paso de simulación, tales como la cantidad de clientes y precios, los cuales se calculan según las reglas o ecuaciones. (5) Al finalizar cada simulación, se consultan las decisiones, es decir los comportamientos del dinero, animales, agua, entre otros.

La simulación del modelo conlleva a espacios de reflexión que ayudan a que los estudiantes reconozcan los procesos de la finca y los recursos comunes como agua, suplemento y a que los clientes que compran sus productos generan variaciones de precios en los productos. Adicionalmente, se aprovechó la ventaja de que el sector finca fue implementado previamente a esta propuesta por Gómez et al. (2020) en un videojuego serio y en un sistema de información, y se les permite a los estudiantes jugar algunas partidas para que comprendan con más detalle la aplicabilidad de los modelos en el desarrollo de software.

\section{Diseño en UML del Software (fase $v$ y vi)}

Para apoyar la fase del díselo en UML del software se especializa el modelo haciendo reconocimiento de tareas presentes cada vez que se genera un evento en la finca. En la figura 8 es presentado el diagrama propuesto, tomado como ejemplo el parto de una vaca preñada, ya que solo esta acción genera otras nueve. 
En la figura se observa parte del diagrama de influencias en gris y se agregan otras en negro con línea a trazos que corresponden con las acciones a controlar, es decir, registrar (1) el parto, requiere (2) ingresar los datos de la cría (nacimiento), (3) su genealogía, (4) el medicamento que le fue suministrado, lo cual (5) actualiza el inventario. Se debe registrar (6) el peso de la cría, la (7) cantidad de leche en el ordeño, el (8) peso de la madre. El software debe generar registro de tareas futuras para (9) inseminar y (10) deslactar la vaca y (11) destetar la cría: El modelo permite estimar los tiempos de las tareas y simula el crecimiento de la cría, por lo tanto, puede usarse como opción de pronóstico para informes de control en reportes de peso.

Para incorporar el desarrollo de software orientado por modelos, se pide a los estudiantes construir el modelo de la finca en UML con una primera versión realizada a mano que después deben pasar a PowerDesigner, una herramienta para el diseño y construcción de software orientado por modelos (Oskaya, 2019). Con los diagramas UML se genera el primer prototipo, valiéndose de la funcionalidad de transformación del diagrama de clases a código para una plataforma y lenguaje de destino deseado, en este caso Netbeans y Java.

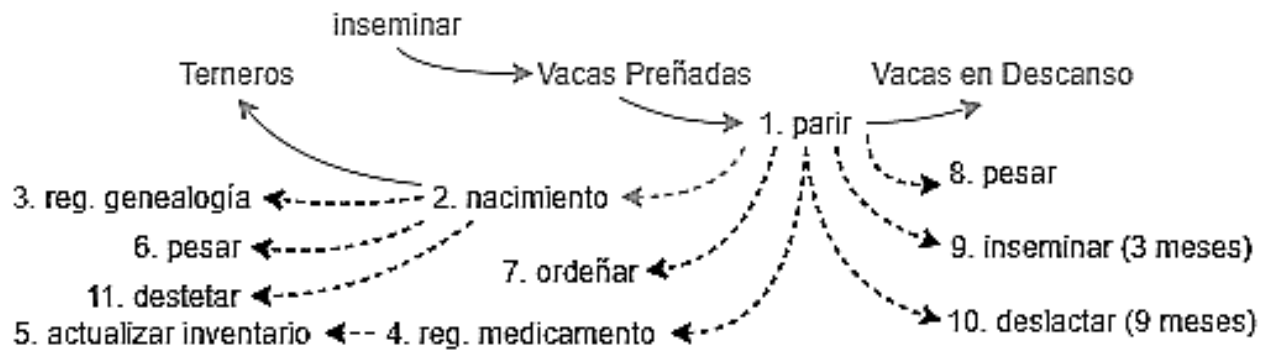

Fig. 8. Ejemplo del reconocimiento de actividades presentes en un proceso generado por el modelo

Como ejemplo se les presenta a los estudiantes el diagrama de casos de uso del profesor, el cual presenta diez funcionalidades que realiza el ganadero y que se deberían controlar con el software: registrar compra, venta o muerte, inseminación, parto, peso, ordeño, suministro de agua, suplemento, medicamento. El estudiante debe comprender que hay procesos internos que actualizan entidades como por ejemplo lo expresado para cada parto. La figura 9 presenta el diagrama de clases, el cual tiene la clase animal (puede ser hembra o macho) con sus atributos y eventos principales; también presenta las relaciones con comercialización que lleva registro de la oferta y demanda con elementos como inventario y precio, y con el registro de agua y suplemento (el diagrama coincide con los subsistemas presentados en la figura 5).

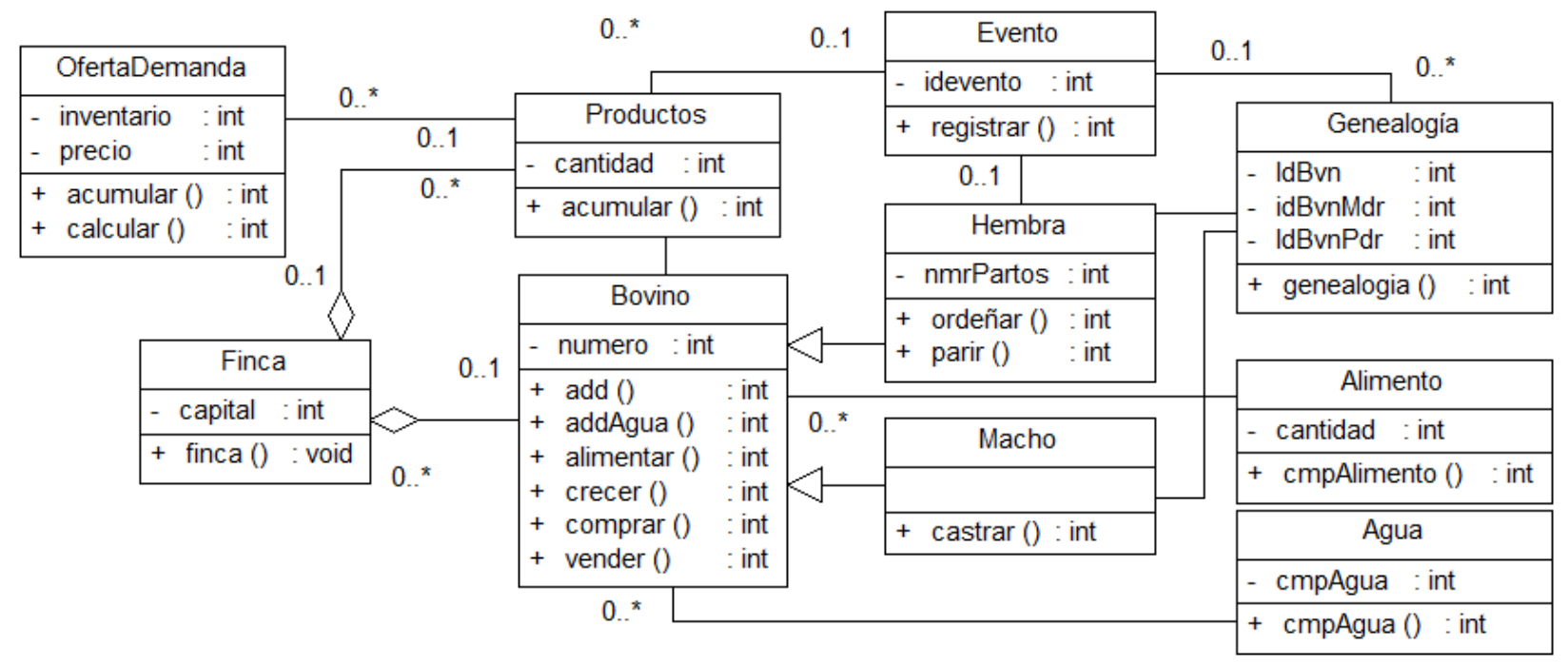

Fig. 9. Diagramas de Clases del software a programar

La figura 10 presenta el Diagrama de Actividades general de operación del software y el del parto. En el primero el proceso inicia tras comprar un animal y continua con el suministro de agua 0 alimento 0 medicamento, la inseminación o la venta. Esas acciones se pueden llevar a cabo en varios periodos de tiempo y requieren cálculos que el software hace y, si es el momento, se informa el parto o un control de sanidad. El segundo diagrama muestra que el evento requiere vistas para indicar la madre y, la cría (la cual genera eventos por realizar) y la genealogía. Tras los primeros diagramas propuestos por los estudiantes de los dos grupos, el conocimiento es homogenizado, la práctica mostró menor comprensión en el grupo de control. La transformación de los modelos a código muestra a los estudiantes que la comprensión mejora el diseño y que buenos diagramas favorecen el desarrollo. De esa manera se acerca al desarrollo de software orientado por 
modelos, competencia que deben desarrollar en el proceso de formación que están iniciando en el programa de Ingeniería de Sistemas. Parte del código generado es presentado en la figura 11, en la cual se aprecia la clase finca (en donde por ejemplo en la línea 23 se define un arreglo de bovinos) y la clase bovino con sus atributos y métodos (en donde por ejemplo entre las líneas 23 y 26 se calcula el alimento).

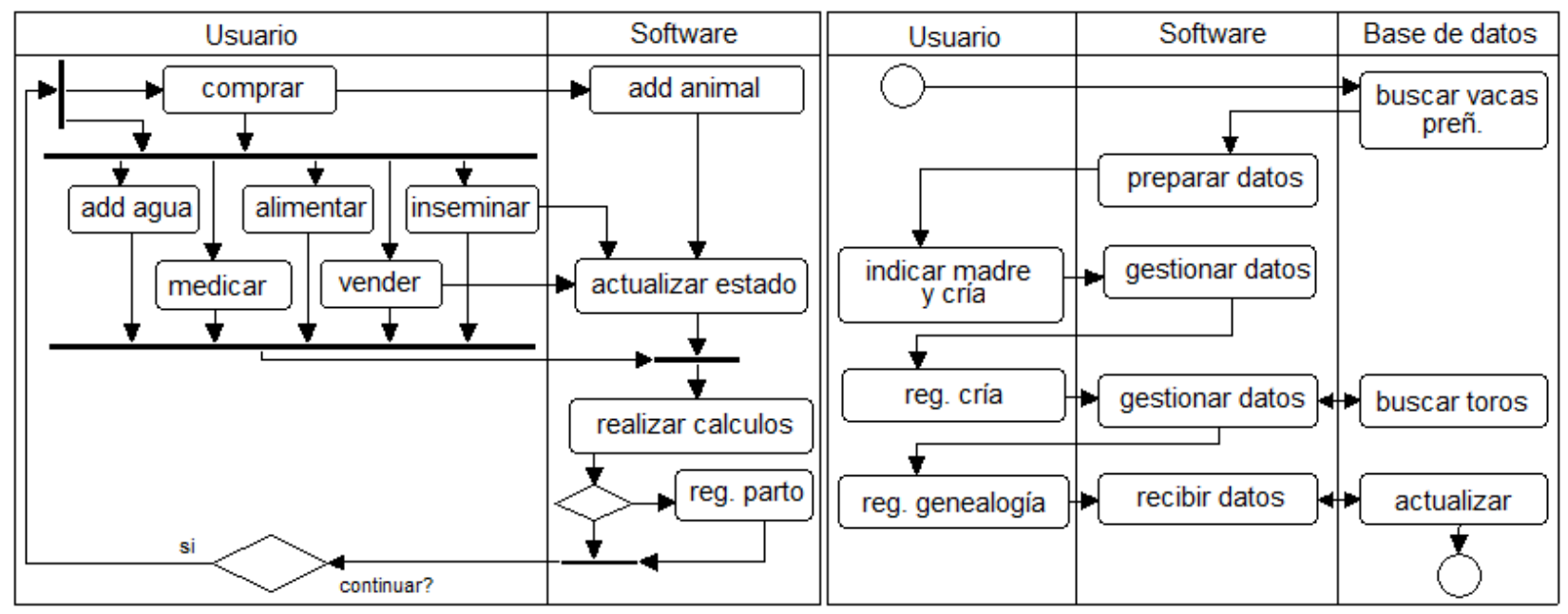

Fig. 10. Diagrama de Actividades del software a programar

\begin{tabular}{|c|c|c|c|c|c|c|c|c|}
\hline \multicolumn{3}{|c|}{ 国 Finca.java $\times$} & $1 D$ & $\nabla \square$ & \multicolumn{4}{|c|}{ 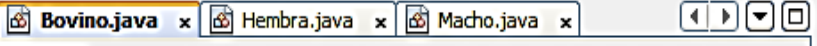 } \\
\hline Source & & -listory & 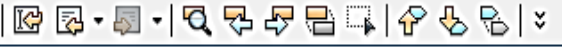 & 田 & Sourc & & 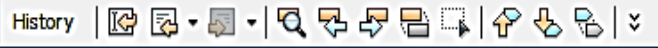 & 田 \\
\hline 5 & & publi & c class Finca \{ & 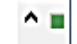 & () & & public class Bovino \{ & $\mathbf{v}$ \\
\hline 6 & & & nt Capital; & & 6 & & public int numero; & \\
\hline 22 & & & nt $\operatorname{cntMxmBvn}=10$; & & 7 & & public int peso; & \\
\hline 23 & & & ovino [ ] bvn = new Bovino [cntMxmBvn]; & - & 8 & & public String raza; & \\
\hline 24 & & & ublic Finca () \{ & & 13 & $\boxplus$ & public int add ()$\{\ldots 6$ lines $\}$ & \\
\hline 25 & & & Capital = 10000000; & & 19 & $\boxplus$ & public int addAgua () $\{\ldots 4$ lines $\}$ & \\
\hline 26 & & & for (int $j=0 ; j<\operatorname{cntMxmBvn;~} j++$ ) \{ & & 23 & 田 & public int alimentar ()$\quad\{\ldots 4$ lines $\}$ & \\
\hline 27 & & & bvn $[j]=$ new Bovino (): & & 27 & $\boxplus$ & public int crecer ()$\quad\{\ldots 4$ lines $\}$ & \\
\hline 28 & & & \} & & 31 & $\bigoplus$ & public int comprar ()$\{\ldots 5$ lines $\}$ & \\
\hline 36 & & \} & & & 36 & $\boxplus$ & public int vender ()$\{\ldots 5$ lines $\}$ & \\
\hline
\end{tabular}

Fig. 11. Código en Java del software diseñado

Esta propuesta es un ejemplo de que el uso de Dinámica de Sistemas permite modelar los procesos de un sistema dinámico en diferentes escenarios (Featherston y Doolan, 2013) y facilita la comprensión y explicación de los procesos presentes y puedan ser tomados por aprendices en desarrollo de software, como base para usarlos en otras actividades aprovechando las siguientes características: i) Muestran las relaciones entre las variables que lo componen y que apoyen experimentos virtuales que simulen situaciones que se pueden presentar en ellos (Wu, 2018); ii) Ayudan a hacer trazabilidad de procesos organizacionales; iii) Se transforman en un diagrama de clases que represente la complejidad del sistema, algo similar a lo realizado por Flórez et al. (2016) para representar un posible observatorio de ciberseguridad; y iv) Permiten reutilizar ecuaciones del modelo para implementar las mecánicas en un videojuego serio o para generar pronósticos en un DSS similar a los presentados por Gómez et al. (2020).

\section{CONCLUSIONES}

La Propuesta de formación dirigida a estudiantes de ingeniería de sistemas ayudó a mejorar la comprensión de la complejidad de los sistemas productivos explicando la importancia de la asociatividad, mostró la Dinámica de Sistemas como apoyo al modelado en UML de esa complejidad y explicó el desarrollo software orientado por modelos, evidencia de ello es que el grupo de estudiantes que utilizó los modelos de simulación, obtuvo mejores resultados en la prueba de conocimientos de los sistemas y diseñó mejores diagramas comparados con respecto a quienes no los usaron ya que se encontró que los integrantes del grupo experimental representaron más interrelaciones en los diagramas de casos de uso, más atributos y métodos en los diagramas de clases y más detalle en los diagramas de actividades. Se espera aplicar la propuesta a una mayor cantidad de estudiantes y en cursos superiores como el de Desarrollo Web o Desarrollo de Videojuegos, e integrar estudiantes de Agroindustria con los Ingeniería de Sistemas para proponer software que mejoren la trazabilidad de procesos y decisiones de ese sector. 


\section{REFERENCIAS}

Andrade, H., Dyner, I., y otros 3 autores, Pensamiento Sistémico, Diversidad en búsqueda de unidad, Editorial UIS, Bucaramanga, Colombia (2001).

Brambilla, M., Cabot, J., y Wimmer, M., Model-driven software engineering in practice, Morgan \& Claypool Publishers, Vermont, United States (2017).

Bunge, M., La Ciencia: su método y su filosofía, Laetoli, Buenos Aires, Argentina (2018).

De Buck, A., Van, I., y Wossink, G., Farmers' reasons for changing to more sustainable practices, https://doi.org/10.1080/13892240108438817, Journal of Agricultural Education and Extension, 7(3), 153-166 (2001).

Featherston, C., y Doolan, M., Using system dynamics to inform scenario planning: a case study, The 31st international conference of the System Dynamics Societ, 53-59, Cambridge, USA, 21-25 de julio (2013).

Flórez, A., Serrano, L., y otros tres autores, Analysis of dynamic complexity of the cyber security Ecosystem of Colombia, https://doi.org/10.3390/fi8030033, Future Internet, 8(3), 33 (2016).

Fonseca, N., González, M., y Narváez, C., Asociatividad para la administración los sistemas de producción campesina, https://doi.org/10.22490/25392786.3644, Estrategia Organizacional, 9(1), 1-17 (2020).

Gómez, O., y Gómez, U., Modelo de simulación para el proceso de producción en empresas de confección textil, https://doi.org/10.18046/syt.v11i24.1503, Sistemas y Telemática, 11(24), 73-89 (2013).

Gómez, O., y Gómez, U., Simulación Cinemática de un robot seguidor de línea para el desarrollo del videojuego de programación Rusty Roads en el Framework Unity, https://doi.org/10.4067/S0718-07642017000500008, Información Tecnológica, 28(5), 55-64 (2017)

Gómez-Prada, U., Orellana-Hernández, M., y Salinas-Ibáñez, J., Apropiación de Sistemas de tecnologías de la información para toma de decisiones de productores agroindustriales basada en videojuegos serios, https://doi.org/10.4067/S0718-07642019000500331, Información Tecnológica, 30(5), 331-340 (2019).

Gómez, U., Andrade, H., y Vásquez, C., Lineamientos Metodológicos para construir Ambientes de Aprendizaje en Sistemas Productivos Agropecuarios soportados en Dinámica de Sistemas, https://doi.org/10.4067/S071807642019000500331, Información Tecnológica, 25 (4), 125-136 (2015).

Gómez, U., Pérez, J., y Ramírez, J., Sistema de Información Agrícola para la disminución de Brechas entre Oferta y Demanda, https://doi.org/10.4067/S0718-07642016000300020, Información Tecnológica, 27(3), 215-220 (2016).

Gómez-Prada, U., Orellana-Hernández, M., y Salinas-lbañez, J., Strategy for the appropriation of a DSS in small bovine producers using simulation and a serious video game, https://doi.org/10.3390/info11120566, Information, 11(12), 566 (2020).

Hurnyak, I., y Kordonska, A., Efficient Use of Common Resources in Conditions of Sustainable Development, https://doi.org/10.12775/TIS.2018.007, Torun International Studies, 1(11), 75-87 (2018).

Jaime, R., Modelamiento semántico con Dinámica de Sistemas en el proceso de desarrollo de software, Revista lbérica de Sistemas e Tecnologías de Informação, 10, 19-33 (2012)

Liebel, G., Marko, N., y otros 3 autores, Assessing the state-of-practice of model-based engineering in the embedded systems domain, https://doi.org/10.1007/978-3-319-11653-2_11, International Conference on Model Driven Engineering Languages and Systems, 166-182, Valencia, Spain, 28-30 de septiembre (2014).

Lince, E., Andrade, H., y Hernández, E., Evolución: herramienta software para modelado y simulación con Dinámica de Sistemas, Revista de Dinámica de Sistemas, 4(1), 1-27 (2011).

Markauskaite, L., Kelly, N., y Jacobson, M., Model-based knowing: How do students ground their understanding about climate systems?, Research in Science Education, 50(1), 53-77 (2020).

O’Connor, J., y McDermott, I., Introducción al Pensamiento Sistémico, Urano, Barcelona, España (1998).

Ozkaya, M., Are the UML modelling tools powerful enough for practitioners? A literature review, https://doi.org/10.1049/iet-sen.2018.5409, IET Software, 13(5), 338-354 (2019).

Sánchez, I., Evaluación de una Renovación Metodológica para un Aprendizaje Significativo de la Física, https://doi.org/10.4067/S0718-50062012000500006, Formación Universitaria, 5(5), 51-65 (2012).

Senge, P., Kleiner, A., y otros 3 autores, The dance of change: The challenges to sustaining momentum in learning organizations, https://doi.org/10.1002/pfi.4140380511, Performance Improvement, 38 (5), 55-58 (2007).

Tulinayo, F., Van Bommel, P., y Proper, H., Grounded system dynamics: A procedure for underpinning system dynamics with a domain modeling method, https://doi.org/10.1007/978-3-642-24849-8_9, Conference on the practice of Enterprise Modeling, 112-125, Oslo, Norway, 2-3 de noviembre (2011).

Vera, P., Nikulin, C., y otros dos autores, Prospective study using archetypes and system dynamics, https://doi.org/10.1108/ARLA-05-2017-0151, Rev. Lat. de Administración, 32(2), 181-202 (2019).

Wu, J., Space Design Teaching Model Using Virtual Simulation Technology, https://doi.org/10.3991/ijet.v13i06.8585, Int. Journal of Emerging Technologies in Learning, 13(6), 163-175 (2018). 